\title{
A potential role for cannabichromene in modulating TRP channels during acute respiratory distress syndrome
} Hesam Khodadadi ${ }^{1,2}$, Évila Lopes Salles ${ }^{1,2}$, Eunice Shin ${ }^{3}$, Abbas Jarrahi $^{4}$, Vincenzo Costigliola ${ }^{5}$, Pritesh Kumar $^{6}$,
Jack C. Yu ${ }^{7}$, John C. Morgan ${ }^{8}$, David C. Hess ${ }^{9}$, Kumar Vaibhav $^{4}$, Krishnan M. Dhandapani $^{4}$ and Babak Baban ${ }^{1,2^{*}}$

\begin{abstract}
Background: Acute respiratory distress syndrome (ARDS) is a life-threatening clinical syndrome whose potential to become one of the most grievous challenges of the healthcare system evidenced by the COVID-19 pandemic. Considering the lack of target-specific treatment for ARDS, it is absolutely exigent to have an effective therapeutic modality to reduce hospitalization and mortality rate as well as to improve quality of life and outcomes for ARDS patients. ARDS is a systemic inflammatory disease starting with the pulmonary system and involves all other organs in a morbid bidirectional fashion. Mounting evidence including our findings supporting the notion that cannabinoids have potential to be targeted as regulatory therapeutic modalities in the treatment of inflammatory diseases. Therefore, it is plausible to test their capabilities as alternative therapies in the treatment of ARDS. In this study, we investigated the potential protective effects of cannabichromene (CBC) in an experimental model of ARDS.
\end{abstract}

Methods: We used, for the first time, an inhalant CBC treatment as a potential therapeutic target in a murine model of ARDS-like symptoms. ARDS was induced by intranasal administration of Poly(l:C), a synthetic mismatched doublestranded RNA, into the C57BL/6 mice (6-10 male mice/group, including sham, placebo, and CBC treated), three once-daily doses followed by a daily dose of inhalant CBC or placebo for the period of 8 days starting the first dose 2 $\mathrm{h}$ after the second Poly(l:C) treatment. We employed histologic, immunohistochemistry, and flow cytometry methods to assess the findings. Statistical analysis was performed by using one way analysis of variance (ANOVA) followed by Newman-Keuls post hoc test to determine the differences among the means of all experimental groups and to establish significance $(p<0.05)$ among all groups.

Results: Our data showed that $\mathrm{CBC}$ was able to reverse the hypoxia (increasing blood $\mathrm{O}_{2}$ saturation by $8 \%$ ), ameliorate the symptoms of ARDS (reducing the pro-inflammatory cytokines by $50 \%$ in lung and blood), and protect the lung tissues from further destruction. Further analysis showed that $C B C$ may wield its protective effects through transient receptor potential (TRP) cation channels, TRPA 1 and TRPV 1 , increasing their expression by 5 -folds in lung tissues compared to sham and untreated mice, re-establishing the homeostasis and immune balance.

Conclusion: Our findings suggest that inhalant $C B C$ may be an effective alternative therapeutic target in the treatment of ARDS. In addition, Increased expression of TRPs cation channels after CBC treatment proposes a novel role for TRPs (TRPA1 and TRPV2) as new potential mechanism to interpret the beneficial effects of CBC as well as other

\footnotetext{
${ }^{*}$ Correspondence: bbaban@augusta.edu

${ }^{2}$ Center for Excellence in Research, Scholarship and Innovation, Dental

College of Georgia, Augusta University, Augusta, GA, USA

Full list of author information is available at the end of the article
}

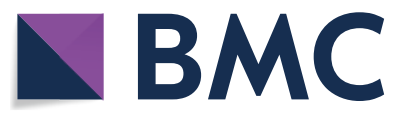

(c) The Author(s) 2021. Open Access This article is licensed under a Creative Commons Attribution 4.0 International License, which permits use, sharing, adaptation, distribution and reproduction in any medium or format, as long as you give appropriate credit to the original author(s) and the source, provide a link to the Creative Commons licence, and indicate if changes were made. The images or other third party material in this article are included in the article's Creative Commons licence, unless indicated otherwise in a credit line to the material. If material is not included in the article's Creative Commons licence and your intended use is not permitted by statutory regulation or exceeds the permitted use, you will need to obtain permission directly from the copyright holder. To view a copy of this licence, visit http://creativecommons.org/licenses/by/4.0/. 
cannabinoids in the treatment of ARDS as well as other inflammatory diseases. Importantly, delivering CBC through an inhaler device is a translational model supporting the feasibility of trial with human subjects, authorizing further research.

Keywords: Cannabichromene, CBC, ARDS, TRPA1, TRPV1, COVID-19

\section{Introduction}

Acute respiratory distress syndrome (ARDS) is a serious complication of sepsis, initiated by cytokine storm due to imbalance of host immunity with exaggerated inflammatory responses (Hu et al., 2020; Matthay et al., 2019). Despite controversy in the definition of ARDS, there is a consensus over the crucial role of Immune response dysregulation in the pathophysiology of ARDS (Matthay et al., 2019; Matthay et al., 2021). As a systemic inflammatory disease, ARDS is initiated by activation of alveolar macrophages, recruitment of neutrophils, and circulating macrophages to the stressed lung tissues. As a consequent of such cellular activations, ARDS advances with elevated level of pro-inflammatory cytokines such as IL-6, IL-17, and IFN $\gamma$, damaging lung tissues and eliciting accumulation of macromolecules manifested by edema and hypoxemia (Han \& Mallampalli, 2015). Contrary to all advancements in understanding the mechanisms and pathophysiology of ARDS, however, no pharmacologic modality for the treatment of ARDS has been yet identified. As evidenced by the COVID-19 pandemic, supportive therapies along with combinatorial therapies such as corticosteroid and IL-6 inhibitor have shown beneficial effects in the management of ARDS (Pan et al., 2018; Harahwa et al., 2020; Catherine, 2014). Therefore, the need to develop alternative novel therapeutic targets for the prevention and treatment of ARDS persists.

Cannabinoids are naturally occurring compounds in Cannabis plants (Atakan, 2012). Numerous studies suggest beneficial effects of cannabinoids in clinical settings (Kogan, 2007; The Health Effects of Cannabis and Cannabinoids: The Current State of Evidence and Recommendations for Research, 2017; Maione et al., 2011). Of over 100 known cannabinoids, four, including tetrahydrocannabinol $\left(\Delta^{9}-\mathrm{THC}\right)$, cannabidiol (CBD), cannabinol $(\mathrm{CBN})$, and cannabichromene $(\mathrm{CBC})$, have attracted the most attention due to supporting evidence of their potential as therapeutic targets (Maione et al., 2011; Izzo et al., 2012; Patil et al., 2020; Martin et al., 2021.; Anderson et al., 2021). Despite their structural differences, both $\mathrm{CBD}$ and $\mathrm{CBC}$ are non-psychoactive compounds with potential immunomodulatory effects and several other health benefits (Maione et al., 2011; Izzo et al., 2012; Pollastro et al., 2018). More recently, in a set of preclinical studies, we showed for the first time that CBD could ameliorate the symptoms of ARDS (Salles et al., 2020;
Khodadadi et al., 2020). Importantly, unlike THC and $\mathrm{CBD}, \mathrm{CBC}$ is not categorized as a scheduled compound by US Drug Enforcement Agency (DEA), which may facilitate the use and assessment of $\mathrm{CBC}$ in clinical settings (BayMedica White paper on Cannabichromene, n.d.). Further, several reports suggest that $\mathrm{CBC}$ may act through the transient receptor potential (TRP) cation channel subfamily A member 1 (TRPA1) as well as transient receptor potential cation channel subfamily $\mathrm{V}$ member 1 (TRPV1) with less affinity. TRPs are involved in vast variety of vital cellular processes, influencing the dynamic of physiologic and immunologic interactions within the multicellular organism (Muller et al., 2019; Froghi et al., 2021; Kun et al., 2014; Tsumura et al., 2012; Romano et al., 2013). CBC binding to TRPs would likely affect tissue homeostasis and immune balance, modulating the inflammatory responses as well as endocannabinoid cellular reuptake (Pollastro et al., 2018; De Petrocellis et al., 2011). CBC has also been reported to be an agonist to the $\mathrm{CB} 2$ receptor, activation of which may further modulate inflammatory responses (Izzo et al., 2012; Udoh et al., 2019).

In this study, we investigated the potential protective effects of $\mathrm{CBC}$, possible mechanisms of action, and whether $\mathrm{CBC}$ can improve symptoms in an experimental model of ARDS.

\section{Materials and methods \\ Animals}

The studies utilized male, 10-12 weeks of age, C57BL/6 mice which were obtained from Jackson Laboratory USA and housed in the laboratory animal facilities of the Augusta University with free access to food and water. These studies conformed to guidelines of Institutional Animal Care and Use Committee.

\section{Experimental design}

ARDS was induced as described previously (Salles et al., 2020; Khodadadi et al., 2020). Briefly, mice were divided into three groups: sham, control, and treatment $(n=$ 6-10) in an open-labeled fashion to explore the efficacy of the treatment. All experiments were performed in accordance with the rules and regulations of the Augusta University Institutional Animal Care and Use Committee (IACUC). All mice were anesthetized with isoflurane. Sham group received phosphate-buffered saline (PBS) 


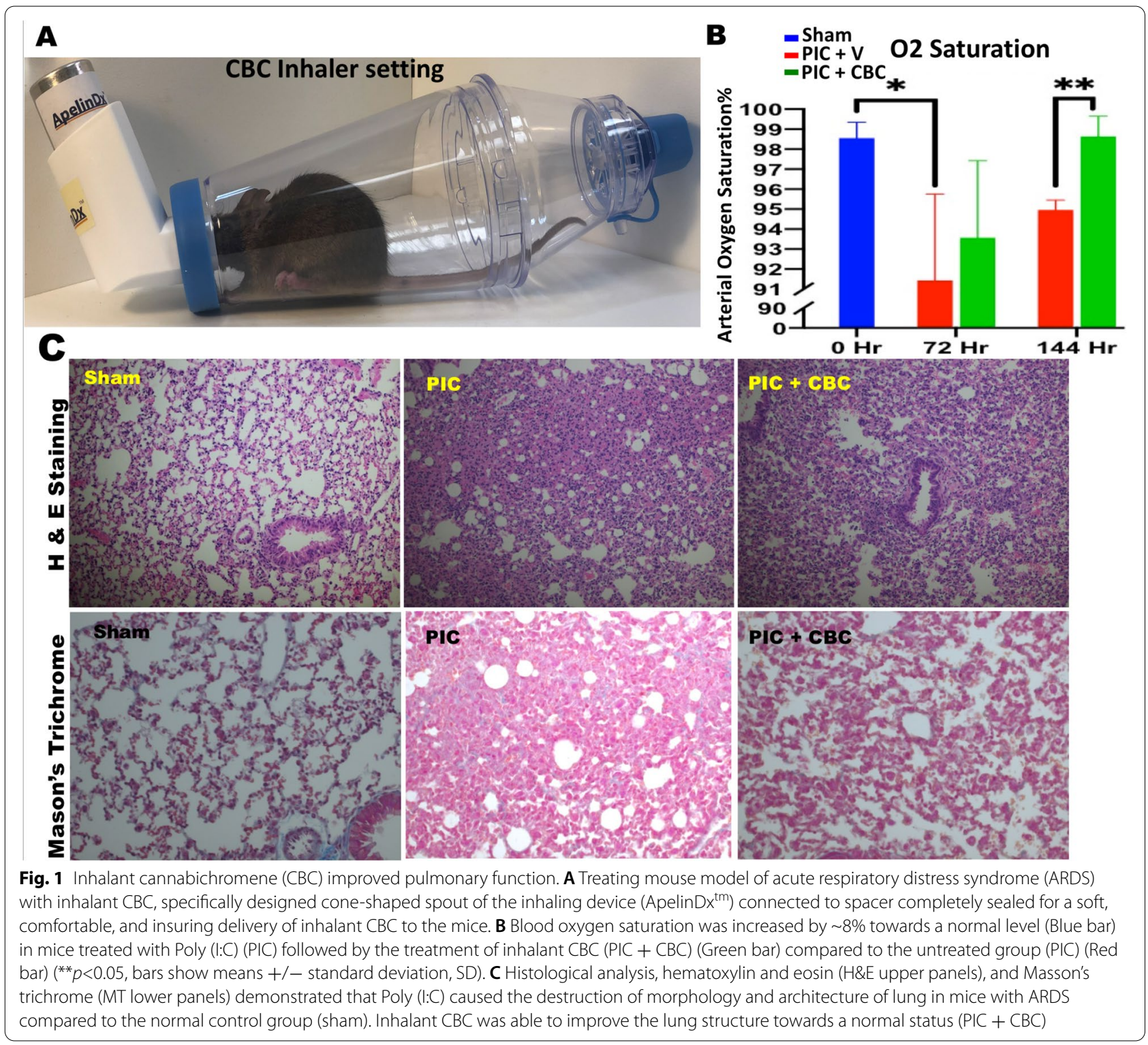

while control and treatment groups were administered Poly(I:C) (Sigma Aldrich, St. Louis, MO, USA) (100 mg in $50 \mathrm{~mL}$ sterile PBS) intranasally (IN) three once-daily doses. CBC was generously provided by BayMedica USA (Prodiol ${ }^{\mathrm{TM}} \mathrm{CBC}$ ) and was administered through an inhaler (ApelinDx ${ }^{\mathrm{tm}}, \mathrm{TM}$ Global Bioscience) $(5 \mathrm{mg} / \mathrm{kg}$, Fig. 1A), first dose $2 \mathrm{~h}$ after the second Poly(I:C) treatment, and every other day for a total of three doses to the treatment group. The dose and administration route of the drug were determined based on previous independent as well as our own studies (Khodadadi et al., 2020; Millar et al., 2019; Borghardt et al., 2018; Rau, 2005). Inhalant delivery provides advantages including local drug delivery with expected minimal side effects, rapid onset and high efficiency impact on the target site and simulating common route of administration seen in clinical scenarios. Sham and control groups received placebo through inhaler carrying the vehicle only. All mice were sacrificed at 8 days after the first Poly(I:C) application. Blood and lung tissues were harvested and subjected to further analysis. Blood oxygen saturation was measured using portable pulse oximeter through carotid arteries, before and after any treatment.

\section{Histology and immunohistochemistry}

Multiple $5 \mu \mathrm{m}$ midcoronal paraffin-embedded sections of lung tissues were cut and stained with hematoxylin and eosin, or trichrome. As for inflammatory indices, 
immunohistochemistry was performed as described previously (Salles et al., 2020; Khodadadi et al., 2020). Briefly, paraffin sections of lung tissues were deparaffinized in xylene and rehydrated by passing the slides through graded alcohol solutions. Endogenous peroxidase was quenched with $3 \% \mathrm{H}_{2} \mathrm{O}_{2}$ in PBS. The sections were then washed in distilled water and heated at $95{ }^{\circ} \mathrm{C}$ in antigen retrieval buffer (Dako, Glostrup, Denmark). Nonspecific staining was blocked with $5 \%$ normal goat serum in PBS for $1 \mathrm{~h}$. Endogenous biotin was inhibited with an avidin/biotin blocking kit (SP2001, Vector Laboratories, Burlingame, $\mathrm{CA}$ ). The sections were then incubated with anti-murine TRPA1, and TRPV1. Preparations were counterstained with hematoxylin and mounted in Faramount and imaged by bright field microscopy. The expression of TRPA1 and TRPV1 were quantified by using particle count and color intensity measurement in the ImageJ software version 1.53 .

\section{Flow cytometry analyses}

For analytical flow cytometry, single-cell suspension was prepared from lung and blood tissues as described previously (Salles et al., 2020; Khodadadi et al., 2020). In brief, tissue samples were sieved through a $100 \mu \mathrm{M}$ cell strainer (BD Biosciences, San Diego, CA, USA), followed by centrifugation $(252 \mathrm{~g}, 10 \mathrm{~min})$ to prepare single-cell suspensions. Then cells were fixed and permeabilized and stained intracellularly for cytokines including IL-6, IL-17, and interferon gamma (IFNY) (proinflammatory cytokines). All samples were run through a 4-Laser LSR
II flow cytometer. Cells were gated based on forward and side scatter properties and on marker combinations to select cells of interest. All acquired flow cytometry data were analyzed using FlowJo V10. Graphs and summary statistics were also used to assess the results.

\section{Statistics}

In order to determine the statistical differences among the means of all experimental groups, data were analyzed using one way analysis of variance (ANOVA) followed by Newman-Keuls post hoc test for multiple comparison and to establish significance $(p<0.05)$ among all groups.

\section{Results}

Inhalant $\mathrm{CBC}$ reversed hypoxia, increasing blood oxygen saturation positively towards the normal level by $8 \%$ from $90 \%$ to $98 \%(+/-0.5 \%)$ (Fig. 1A, B). Histological examination of lung tissues, hematoxylin and eosin staining (Fig. 1C upper panels), and Masson's trichrome staining (Fig. 1C lower panels) demonstrated that $\mathrm{CBC}$ treatment reduced the ARDS-like lung destruction, improved the structural damages to the lung. Further histological examination, immunohistochemistry showed that $\mathrm{CBC}$ treatment increased the level of expression for transient receptor potential (TRP) channels of ankyrin type-1 (TRPA1) and vanilloid type-1 (TRPV1), suggesting an active interaction between CBC and TRPs (Fig. 2). Flow cytometry analysis of blood and lung demonstrated that CBC treatment curtailed the expression levels of proinflammatory cytokines including IL-6, IL-17, and IFNy

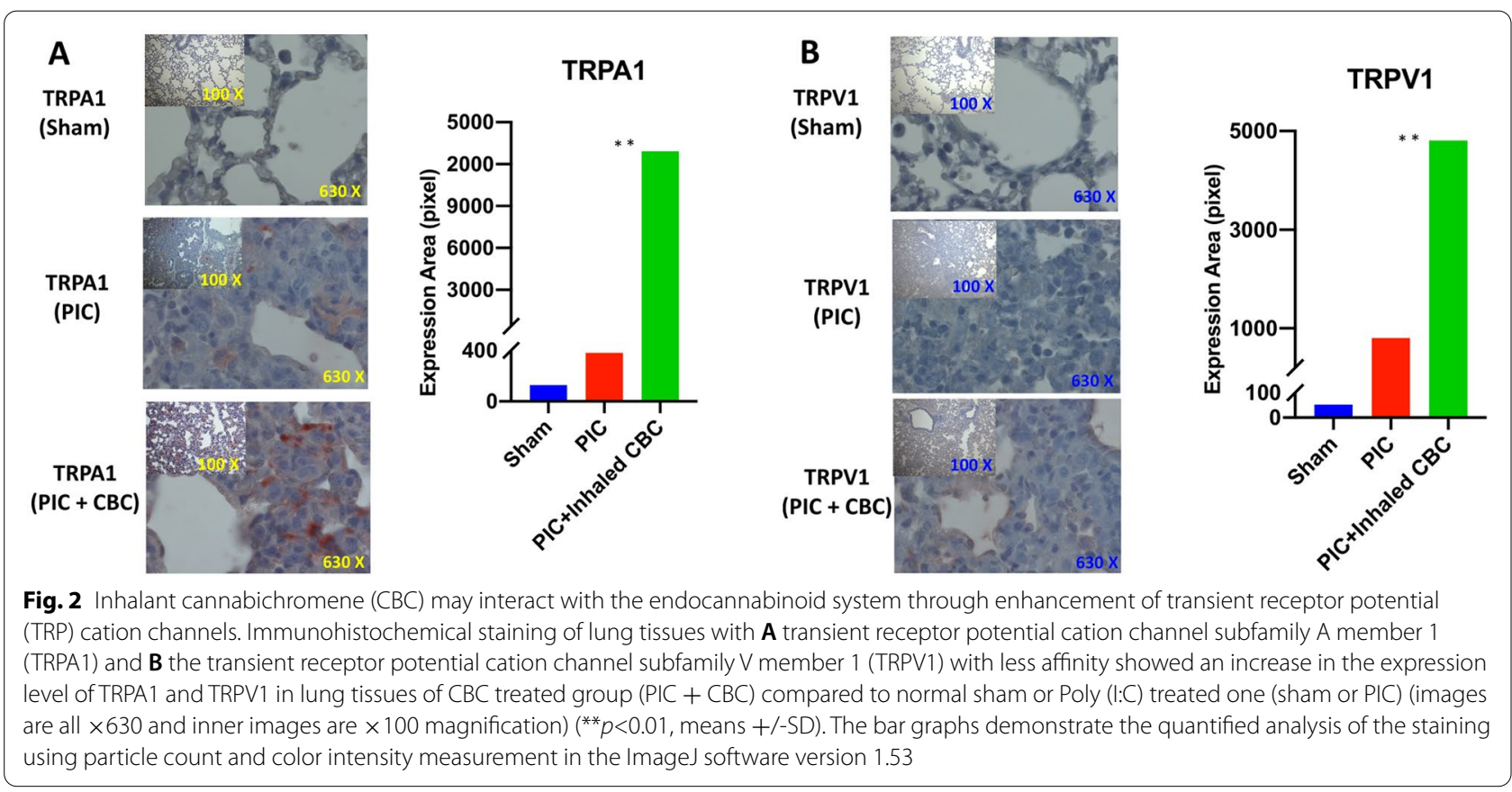



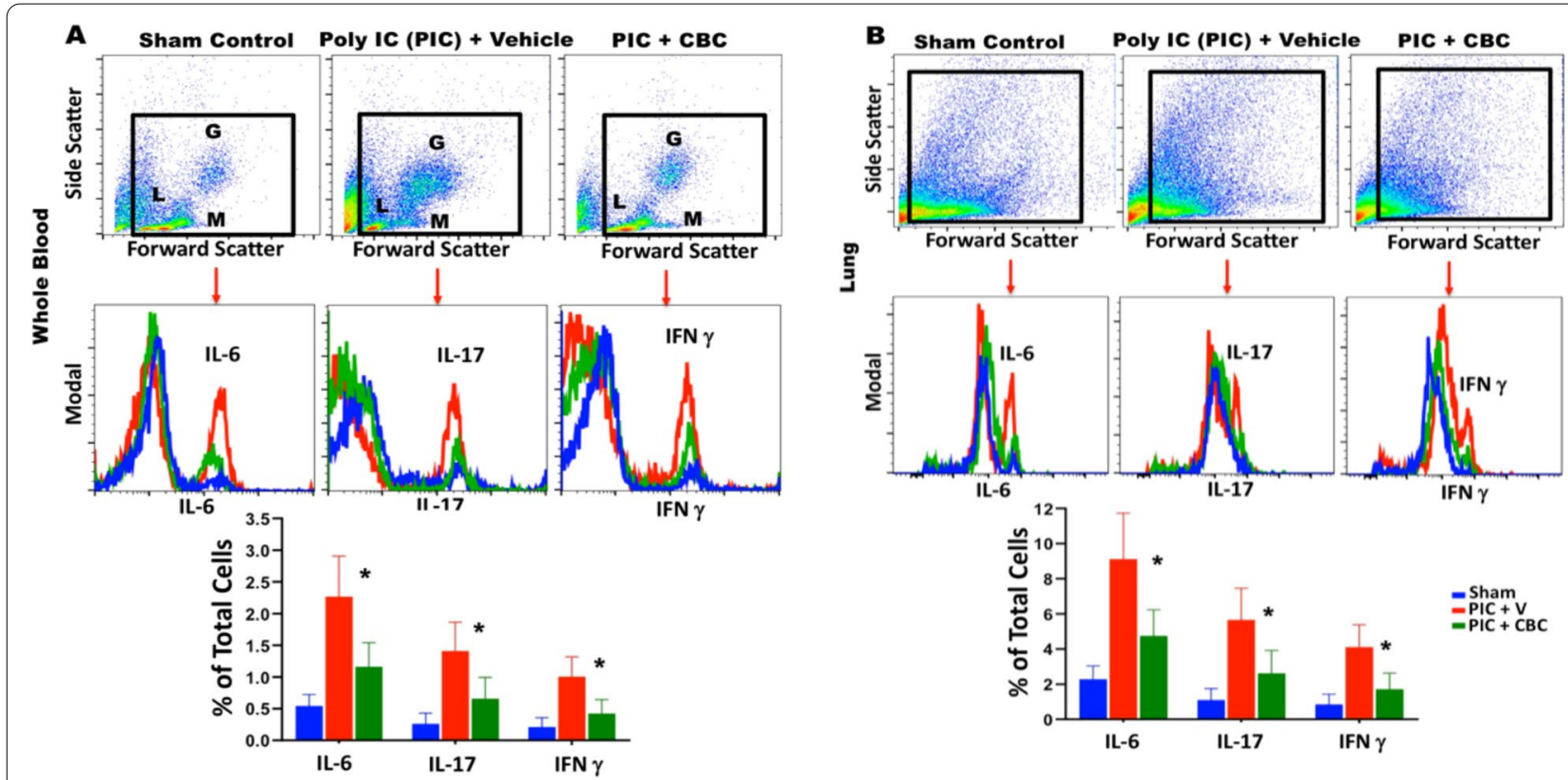

Fig. 3 Inhalant cannabichromene (CBC) reduced ARDS-like induced inflammatory indices. Flow cytometry analysis of $\mathbf{A}$ whole blood and $\mathbf{B}$ lung tissues showed that Intranasal administration of Poly(l:C) effectuated a significant increase of pro-inflammatory cytokines including IL-6, IL-17, and IFNY (PIC group) compared to the control group (sham). These effects were reversed with inhalant CBC (PIC + CBC), resulted in marked downregulation of all three pro-inflammatory cytokines ( ${ }^{*} p<0.03$, means $+/-$ SD). Further, the flow cytometry analysis of whole blood in the PIC group showed increased granulocytes $(G)$ and monocytes $(M)$ while lymphocytes were decreased compared to mice treated with inhalant $C B C$ $(P I C+C B C)$ supporting the notion that inhalant $C B C$ can reverse the adverse effects of ARDS and re-establish the immune balance, resolving lymphopenia and restoring homeostasis

in both tissues compared to the untreated counterparts $(p<0.01)$ (Fig. 3A, B). The histograms show the quantified level of expression of IL-6, IL-17, and IFN $\gamma$ in both tissues treated with $\mathrm{CBC}$ compared to the control sham (untreated with $\mathrm{CBC}$ ) group.

\section{Discussion}

Our findings demonstrated, for the first time, that inhalant $\mathrm{CBC}$ protected lung structure, contained excessive cytokine production, and curtailed inflammatory responses both in lung and blood tissues in an experimental ARDS model. These results were in consistent with findings by other independent studies demonstrating counter-inflammatory effects of CBC in LPS-induced paw edema (DeLong et al., 2010). In addition, CBC is a non-psychoactive cannabinoid which is not categorized as a scheduled compound by US Drug Enforcement Agency (DEA). This may facilitate the use and assessment of CBC in clinical settings (BayMedica White paper on Cannabichromene, n.d.). More importantly, using inhalers for the first time in a therapeutic setting would provide an effective and safer way to deliver $\mathrm{CBC}$ to small airways, lowering required doses and reducing any potential systemic adversarial symptom in a more cost-effective way. Additionally, delivery of $\mathrm{CBC}$ through an inhaler increases the translational values of this study and potential for trials in a clinical setting with implications for the treatment of respiratory complications such as development of ARDS in severe cases of COVID-19 and several respiratory diseases. It is noteworthy that we exclusively employed male animals in our studies to prevent additional confounding factors based on gender differences (e.g., different stages of estrous cycle, hormonal effects), staying focused on the proof of concept for CBC sole effects in ARDS. However, increasing evidence suggests a crucial role for sex dimorphism in occurrence and outcomes of ARDS, warranting further investigations at the pre-clinical level as well as for the potential human clinical trials in the future (Scully et al., 2020).

Further, our data showed that $\mathrm{CBC}$ can increase the expression level of transient receptor potential ankyrin 1 (TRPA1) and vanilloid receptor 1 (TRPV1), supporting the notion of potential interactions between $\mathrm{CBC}$ and TRPA 1 as well as TRPV1 (TRPs). The TRP channels superfamily play vital roles in a wide range of physiological and immunologic processes such as cellular polarization, cytokine production, phagocytosis, and cytotoxicity (Muller et al., 2019; Froghi et al., 2021; Kun et al., 2014; Tsumura et al., 2012). Therefore, it is very plausible to hypothesize that binding CBC to TRPs may 
downregulate the inflammatory responses by shifting the so-called "cytokine storm" to a "cytokine breeze," re-establishing the homeostasis and preventing additional structural damages to the vital organs. Although the interaction between CBC and TRPs has been previously reported (Maione et al., 2011; De Petrocellis et al., 2011), to the best of our knowledge, this is the first report to show the beneficial effects of cross-talk between CBC and TRPs in ARDS. In addition, since several studies have indicated that $\mathrm{CBC}$ may bind to and activate $\mathrm{CB} 2$ receptor of the endocannabinoid system, suggesting a potential synergy between TRPs and CB2 activation as a potential mechanism contributing to the beneficial effects of $\mathrm{CBC}$ in ARDS. In fact, such potential engagement of the endocannabinoid system by CBC may establish a synergistic relationship between exogenous phytocannabinoids $(\mathrm{CBC})$ and endogenous endocannabinoids (e.g., anandamide and 2-AG). Such bi-directional dynamic would have the potential as a therapeutic modality in the treatment of ARDS as well as a wide variety of inflammatory diseases, neurodegenerative diseases, pain control, cancer, chronic wounds, and sleeping disorders.

\section{Conclusion}

These novel findings render a new therapeutic role for $\mathrm{CBC}$ in the treatment of ARDS as well as a wide range of respiratory diseases including a potential for COVID19 and other inflammatory conditions. The use of inhalant $\mathrm{CBC}$ and direct delivery of $\mathrm{CBC}$ to the lungs offers a more rapid onset of action, allows smaller doses to be used, and has a better efficacy to safety ratio compared to systemic therapy. Further, as TRPs are emerging as novel therapeutic targets through their potential roles in cellular interactions and tissue homeostasis (Zhao et al., 2021), therefore, it is plausible to envision more prominent roles for $\mathrm{CBC}$ in tissue homeostasis, modulation of inflammatory responses, and orchestrating a set of cellular interactions as an immunotherapeutic target, warranting further research.

\section{Abbreviations \\ ARDS: Acute respiratory distress syndrome; CBC: Cannabichromene; CBD: Cannabidiol; CBN: Cannabinol; DEA: Drug Enforcement Agency; IL-6: Interleukin-6; IL-17: Interleukin-17; IFNy: Interferon gamma; LPS: Lipopolysac- charides; PIC: Poly I:C; PBS: Phosphate-buffered saline; TRP: Transient receptor potential; TRPA1: Transient receptor potential ankyrin 1; TRPV1:Transient receptor potential vanilloid receptor 1; 2-AG: 2-Arachidonoylglycerol; $\triangle 9$-THC: Tetrahydrocannabinol.}

\section{Acknowledgements}

Authors would like to thank BayMedica (BayMedica.com) for the generous supply of CBC for this study.

\section{Authors' contributions}

$H K, E L S, E S, A J, K V$, and $B B$ performed experiments and analyzed the data. $H K$ and $\mathrm{BB}$ performed statistical analysis. $\mathrm{BB}, \mathrm{KMD}$, and $\mathrm{HK}$ wrote the manuscript.
VC, PK, JCY, JCM, DCH, KMD, and BB contributed to the conceptualization and editing of the text. All authors read and approved the final manuscript.

\section{Funding}

This work was partially supported by funding provided by TM Global Bioscience, LLC, a medically-focused division of ThriftMaster Global Holdings., as well as by awards from the NIH (NS1 10378 to KMD/BB, and NS114560 to KV).

\section{Availability of data and materials}

The datasets used and/or analyzed during the current study are available from the corresponding author on reasonable request.

\section{Declarations}

\section{Ethics approval and consent to participate}

The experiments, materials, and methods were performed in accordance with the rules and regulations of the Augusta University Institutional Animal Care and Use Committee (IACUC).

\section{Consent for publication \\ Not applicable}

\section{Competing interests}

The authors declare that they have no competing interests. Thriftmaster Holding Group (THG) is the provider of inhalers and BayMedica as the provider of CBC which have a contract with Augusta University. THG and BayMedica had no role in study design, data collection and analysis, decision to publish, or preparation of the manuscript.

\section{Author details}

${ }^{1}$ Department of Oral Biology and Diagnostic Sciences, Dental College of Georgia, Augusta University, Augusta, GA, USA. ${ }^{2}$ Center for Excellence in Research, Scholarship and Innovation, Dental College of Georgia, Augusta University, Augusta, GA, USA. ${ }^{3}$ Medical College of Georgia, Augusta University, Augusta, GA, USA. ${ }^{4}$ Department of Neurosurgery, Medical College of Georgia, Augusta University, Augusta, GA, USA. ${ }^{5}$ European Medical Association (EMA), Brussels, Belgium. ${ }^{6}$ Cannabinoid Research Program, Canadore College, North Bay, Ontario, Canada. ${ }^{7}$ Department of Surgery, Medical College of Georgia, Augusta University, Augusta, GA, USA. ${ }^{8}$ Parkinson's Foundation Center of Excellence, Movement Disorders, Program, Department of Neurology, Medical College of Georgia, Augusta University, Augusta, GA, USA. ${ }^{9}$ Department of Neurology, Medical College of Georgia, Augusta University, Augusta, GA, USA.

Received: 19 May 2021 Accepted: 15 September 2021

Published online: 01 October 2021

\section{References}

Anderson LL, Ametovski A, Lin Luo J, Everett-Morgan D, McGregor IS, Banister SD, et al. Cannabichromene, Related Phytocannabinoids, and 5-Fluorocannabichromene Have Anticonvulsant Properties in a Mouse Model of Dravet Syndrome. ACS Chem Neurosci. 2021;12(2):330-9.

Atakan Z. Cannabis, a complex plant: different compounds and different effects on individuals. Ther Adv Psychopharmacol. 2012;2(6):241-54.

Chris Meiering., Prodiol CBC: Cannabichromene Whitepaper, Baymedica Inc, USA., 2021. p. 2. (baymedica.com).

Borghardt JM, Kloft C, Sharma A. Inhaled Therapy in Respiratory Disease: The Complex Interplay of Pulmonary Kinetic Processes. Can Respir J. 2018;2018:2732017.

Catherine L. Hough, Should we ever give steroids to ARDS patients? Clin Chest Med. 2014;35(4):781-95.

De Petrocellis L, Ligresti A, Moriello AS, Allarà M, Bisogno T, Petrosino S, et al. Effects of cannabinoids and cannabinoid-enriched Cannabis extracts on TRP channels and endocannabinoid metabolic enzymes. Br J Pharmacol. 2011;163(7):1479-94.

DeLong GT, Wolf CE, Poklis A, Lichtman AH. Pharmacological evaluation of the natural constituent of Cannabis sativa, cannabichromene and its modulation by $\Delta(9)$-tetrahydrocannabinol. Drug Alcohol Depend. 2010;112(1-2):126-33. 
Froghi S, Grant CR, Tandon R, Quaglia A, Davidson B, Fuller B. New Insights on the Role of TRP Channels in Calcium Signalling and Immunomodulation: Review of Pathways and Implications for Clinical Practice. Clin Rev Allergy Immunol. 2021;60:271-92.

Han S, Mallampalli RK. The acute respiratory distress syndrome: from mechanism to translation. J Immunol. 2015;194(3):855-60.

Harahwa TA, Khan IH, Harky A. Ventilatory support for COVID-19 patients. Acta Biomed. 2020;91(4):e2020122.

Hu Q, Hao C, Tang S. From sepsis to acute respiratory distress syndrome (ARDS): emerging preventive strategies based on molecular and genetic researches. Biosci Rep. 2020;40(5):BSR20200830.

Izzo AA, Capasso R, Aviello G, Borrelli F, Romano B, Piscitelli F, et al. Inhibitory effect of cannabichromene, a major non-psychotropic cannabinoid extracted from Cannabis sativa, on inflammation-induced hypermotility in mice. Br J Pharmacol. 2012;166(4):1444-60.

Khodadadi H, Salles ÉL, Jarrahi A, Chibane F, Costigliola V, Yu JC, et al. Cannabidiol Modulates Cytokine Storm in Acute Respiratory Distress Syndrome Induced by Simulated Viral Infection Using Synthetic RNA. Cannabis Cannabinoid Res. 2020;5(3):197-201.

Kogan NM. Cannabinoids in health and disease. Dialogues Clin Neurosci. 2007;9(4):413-30.

Kun J, Szitter I, Kemény A, Perkecz A, Kereskai L, Pohóczky K, et al. Upregulation of the transient receptor potential ankyrin 1 ion channel in the inflamed human and mouse colon and its protective roles. PLoS One. 2014;9(9):e108164.

Maione S, Piscitelli F, Gatta L, Vita D, De Petrocellis L, Palazzo E, et al. Nonpsychoactive cannabinoids modulate the descending pathway of antinociception in anaesthetized rats through several mechanisms of action. $\mathrm{Br}$ J Pharmacol. 2011;162(3):584-96.

Martin LJ, Cairns EA, Heblinski M, Fletcher C, Krycer JR, Arnold JC, et al. Cannabichromene and $\triangle^{9}$-Tetrahydrocannabinolic Acid Identified as Lactate Dehydrogenase-A Inhibitors by in Silico and in Vitro Screening. J Nat Products. 2021:84(5):1469-77.

Matthay MA, Thompson BT, Ware LB. The Berlin definition of acute respiratory distress syndrome: should patients receiving high-flow nasal oxygen be included? Lancet Respir Med. 2021;26 S2213-2600(21)00105-3.

Matthay MA, Zemans RL, Zimmerman GA, Arabi YM, Beitler JR, Mercat A, et al. Acute respiratory distress syndrome. Nat Rev Dis Primers. 2019;5(1):18.

Millar SA, Stone NL, Bellman ZD, Yates AS, England TJ, O'Sullivan SE. A systematic review of cannabidiol dosing in clinical populations. Br J Clin Pharmacol. 2019;85(9):1888-900
Muller C, Morales P, Reggio PH. Cannabinoid Ligands Targeting TRP Channels. Front Mol Neurosci. 2019;11:487.

Pan C, Liu L, Xie J-F, Qiu H-B. Acute Respiratory Distress Syndrome: Challenge for Diagnosis and Therapy. Chin Med J (Engl). 2018;131(10):1220-4.

Patil AS, Mahajan UB, Agrawal YO, Patil KR, Patil CR, Ojha S, et al. Plant-derived natural therapeutics targeting cannabinoid receptors in metabolic syndrome and its complications: A review. Biomed Pharmacother. 2020;132:110889.

Pollastro F, Caprioglio D, Del Prete D, et al. Cannabichromene. Nat Product Commun. 2018;13(9):1189-94.

Rau JL. The inhalation of drugs: advantages and problems. Respir Care. 2005;50(3):367-82.

Romano B, Borrelli F, Fasolino I, Capasso R, Piscitelli F, Cascio M, et al. The cannabinoid TRPA1 agonist cannabichromene inhibits nitric oxide production in macrophages and ameliorates murine colitis. Br J Pharmacol. 2013;169(1):213-29.

Salles ÉL, Khodadadi H, Jarrahi A, Ahluwalia M, Paffaro VA Jr, Costigliola V, et al. Cannabidiol (CBD) modulation of apelin in acute respiratory distress syndrome. J Cell Mol Med. 2020;24(21):12869-72.

Scully EP, Haverfield J, Ursin RL, Tannenbaum C, Klein SL. Considering how biological sex impacts immune responses and COVID-19 outcomes. Nat Rev Immunol. 2020;20(7):442-7.

The Health Effects of Cannabis and Cannabinoids: The Current State of Evidence and Recommendations for Research., Committee on the Health Effects of Marijuana: An Evidence Review and Research Agenda. Washington (DC): National Academies Press (US); 2017.

Tsumura M, Sobhan U, Muramatsu T, Sato M, Ichikawa H, Sahara Y, et al. TRPV1-mediated calcium signal couples with cannabinoid receptors and sodium-calcium exchangers in rat odontoblasts. Cell Calcium. 2012;52(2):124-36.

Udoh M, Santiago M, Devenish S, McGregor IS, Connor M. Cannabichromene is a cannabinoid CB2 receptor agonist. Br J Pharmacol. 2019;176(23):4537-47.

Zhao Y, McVeigh BM, Moiseenkova-Bell VY. Structural pharmacology of TRP channels. Rev J Mol Biol. 2021;166914.

\section{Publisher's Note}

Springer Nature remains neutral with regard to jurisdictional claims in published maps and institutional affiliations.
Ready to submit your research? Choose BMC and benefit from:

- fast, convenient online submission

- thorough peer review by experienced researchers in your field

- rapid publication on acceptance

- support for research data, including large and complex data types

- gold Open Access which fosters wider collaboration and increased citations

- maximum visibility for your research: over $100 \mathrm{M}$ website views per year

At $\mathrm{BMC}$, research is always in progress.

Learn more biomedcentral.com/submissions 